\title{
A Spectroscopic and Quantum Chemical Study of Cyclopropylmethylphosphine, a Candidate for Intramolecular Hydrogen Bonding
}

(Supporting Information)

George C. Cole, ${ }^{\text {a Harald Møllendal }}{ }^{*, a}$ and Jean-Claude Guillemin ${ }^{\mathrm{b}}$

Department of Chemistry, University of Oslo, P.O. Box 1033 Blindern, NO-0315 Oslo, Norway, Laboratoire de Synthèse et Activation de Biomolécules, UMR CNRS 6052, Institut de Chimie de Rennes, ENSCR, F-35700 Rennes, France

*Corresponding author. E-mail: harald.mollendal@kjemi.uio.no. Tel +47 228556 74, Fax: +4722 855441 .

${ }^{a}$ University of Oslo

${ }^{\mathrm{b}}$ ENSCR 
Table 1S: Microwave Spectrum and Spectroscopic Constants of the Ground Vibrational State of Cyclopropylmethylphosphine, Conformer III

TOTAL NUMBER OF ACCEPTED TRANSITIONS: 123

\begin{tabular}{|c|c|c|c|c|c|c|c|c|c|}
\hline \multicolumn{6}{|c|}{ TRANSITION } & \multirow{2}{*}{\multicolumn{2}{|c|}{$\begin{array}{l}\text { OBS. FREQ. O.-C. } \\
\text {. FREQ. DELTA }\end{array}$}} & \multirow{2}{*}{$\begin{array}{c}\text { DISTORTION } \\
\text { TOTAL }\end{array}$} & \multirow{2}{*}{$\begin{array}{l}\text { CONTRIBUTION } \\
\text { SEXTIC }\end{array}$} \\
\hline$(\mathrm{J}, \mathrm{K}-$ & $\mathrm{K}$ & +1 & & $\mathrm{~K}-1$ & 1, $\mathrm{K}+1)$. & & & & \\
\hline 3 & $\odot$ & 3 & 4 & 1 & 3 & 25005.670 & -0.086 & -0.19 & \\
\hline 4 & $\odot$ & 4 & 5 & 1 & 4 & 29215.640 & -0.042 & -0.37 & \\
\hline 5 & 1 & 4 & 6 & 1 & 5 & $23341.07 \odot$ & $\odot .053$ & $-\odot .61$ & \\
\hline 5 & 2 & 3 & 6 & 2 & 4 & 22957.150 & -0.225 & $-\odot .38$ & \\
\hline 5 & 2 & 4 & 6 & 2 & 5 & 22889.110 & -0.019 & -0.34 & \\
\hline 6 & 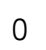 & 6 & 7 & 0 & 7 & 26605.040 & $-\odot .035$ & -0.82 & \\
\hline 6 & 0 & 6 & 7 & 1 & 6 & 37907.940 & $\odot .201$ & -1.12 & \\
\hline 6 & 1 & 5 & 7 & 1 & 6 & 27222.850 & -0.155 & $-\odot .97$ & \\
\hline 6 & 2 & 4 & 7 & 2 & 5 & 26807.450 & $-\odot .051$ & $-\odot .70$ & \\
\hline 6 & 2 & 5 & 7 & 2 & 6 & 26698.650 & -0.152 & -0.63 & \\
\hline 6 & 3 & 3 & 7 & 3 & 4 & 26731.440 & -0.186 & -0.35 & \\
\hline 6 & 3 & 4 & 7 & 3 & 5 & 26729.540 & -0.114 & $-\odot .35$ & \\
\hline 6 & 4 & 2 & 7 & 4 & 3 & 26724.360 & -0.158 & 0.07 & \\
\hline 6 & 4 & 3 & 7 & 4 & 4 & 26724.360 & -0.146 & $\odot .07$ & \\
\hline 6 & 5 & 1 & 7 & 5 & 2 & 26722.120 & -0.128 & 0.61 & \\
\hline 6 & 5 & 2 & 7 & 5 & 3 & 26722.120 & -0.128 & 0.61 & \\
\hline 6 & 6 & $\odot$ & 7 & 6 & 1 & 26721.380 & -0.058 & 1.27 & \\
\hline 6 & 6 & 1 & 7 & 6 & 2 & 26721.380 & $-\odot .058$ & 1.27 & \\
\hline 7 & 0 & 7 & 8 & $\odot$ & 8 & 30367.890 & 0.133 & -1.21 & \\
\hline 7 & $\odot$ & 7 & 8 & 1 & 7 & 42403.740 & $\odot . \odot 81$ & -1.75 & \\
\hline 7 & 1 & 6 & 8 & 1 & 7 & 31101.020 & $\odot .025$ & -1.46 & \\
\hline 7 & 2 & 5 & 8 & 2 & 6 & 30668.170 & 0.143 & -1.14 & \\
\hline 7 & 2 & 6 & 8 & 2 & 7 & 30506.140 & $\odot .052$ & -1.02 & \\
\hline 7 & 3 & 4 & 8 & 3 & 5 & 30555.740 & -0.138 & $-\odot .72$ & \\
\hline 7 & 3 & 5 & 8 & 3 & 6 & 30552.080 & 0.143 & -0.71 & \\
\hline 7 & 4 & 3 & 8 & 4 & 4 & 30544.640 & $\odot .018$ & -0.23 & \\
\hline 7 & 4 & 4 & 8 & 4 & 5 & 30544.640 & 0.052 & -0.23 & \\
\hline 7 & 5 & 2 & 8 & 5 & 3 & 30541.100 & $\odot .063$ & 0.39 & \\
\hline 7 & 5 & 3 & 8 & 5 & 4 & 30541.100 & $\odot .064$ & 0.39 & \\
\hline 8 & 3 & 5 & 9 & 3 & 6 & 34382.640 & 0.006 & -1.21 & \\
\hline 8 & 3 & 6 & 9 & 3 & 7 & 34375.480 & 0.058 & -1.20 & \\
\hline 8 & 4 & 4 & 9 & 4 & 5 & 34365.700 & $\odot .038$ & -0.66 & \\
\hline 8 & 4 & 5 & 9 & 4 & 6 & 34365.700 & 0.121 & -0.66 & \\
\hline 8 & 5 & 3 & 9 & 5 & 4 & 34360.390 & $\odot .035$ & $\odot .04$ & \\
\hline 8 & 5 & 4 & 9 & 5 & 5 & 34360.390 & $\odot .036$ & 0.04 & \\
\hline 9 & $\odot$ & 9 & 10 & $\odot$ & 10 & 37850.110 & -0.052 & -2.28 & \\
\hline 9 & 1 & 8 & 10 & $\odot$ & 10 & 24956.210 & -0.156 & -1.35 & \\
\hline 9 & 0 & 9 & 10 & 1 & 9 & 51735.660 & -0.131 & -3.76 & \\
\hline 9 & 0 & 9 & 10 & 1 & 9 & 51735.660 & -0.131 & -3.76 & \\
\hline 10 & 1 & 9 & 11 & $\odot$ & 11 & $27683.20 \odot$ & -0.125 & -1.48 & \\
\hline 11 & 1 & 10 & 12 & $\odot$ & 12 & 30253.090 & $-\odot .019$ & -1.50 & \\
\hline 12 & 1 & 11 & 12 & 2 & 11 & 21159.640 & $\odot .059$ & 4.22 & \\
\hline 12 & 1 & 11 & 13 & $\odot$ & 13 & 32659.370 & $-\odot .029$ & -1.35 & \\
\hline
\end{tabular}




\begin{tabular}{|c|c|c|c|c|c|c|c|c|c|}
\hline 12 & 2 & 10 & 13 & 1 & 12 & 27370.260 & -0.115 & -8.37 & \\
\hline 12 & 2 & 10 & 13 & 2 & 11 & 50137.830 & $\odot .237$ & -5.98 & \\
\hline 12 & 2 & 11 & 13 & 2 & 12 & 49495.070 & -0.106 & -4.98 & \\
\hline 13 & 1 & 12 & 13 & 2 & 12 & 20251.730 & 0.042 & 5.37 & \\
\hline 12 & 3 & 9 & 13 & 3 & 10 & 49726.230 & 0.119 & -4.84 & \\
\hline 12 & 3 & 10 & 13 & 3 & 11 & 49679.930 & 0.030 & -4.73 & \\
\hline 12 & 4 & 8 & 13 & 4 & 9 & 49661.560 & -0.276 & -3.95 & \\
\hline 12 & 4 & 9 & 13 & 4 & 10 & 49661.560 & 0.906 & -3.94 & \\
\hline 12 & 5 & 7 & 13 & 5 & 8 & 49644.380 & 0.126 & -2.92 & \\
\hline 12 & 5 & 8 & 13 & 5 & 9 & 49644.380 & 0.141 & -2.92 & \\
\hline 12 & 6 & 6 & 13 & 6 & 7 & 49636.260 & 0.195 & -1.68 & \\
\hline 12 & 6 & 7 & 13 & 6 & 8 & 49636.260 & 0.195 & -1.68 & \\
\hline 13 & 1 & 12 & 14 & $\odot$ & 14 & 34898.150 & -0.224 & -1.00 & \\
\hline 13 & 2 & 11 & 14 & 2 & 12 & 54059.650 & 0.160 & -7.61 & \\
\hline 13 & 2 & 12 & 14 & 2 & 13 & 53281.400 & 0.089 & -6.27 & \\
\hline 13 & 3 & 10 & 14 & 3 & 11 & 53574.610 & 0.038 & -6.27 & \\
\hline 13 & 3 & 11 & 14 & 3 & 12 & 53507.690 & -0.097 & -6.08 & \\
\hline 13 & 4 & 9 & 14 & 4 & 10 & 53489.690 & 0.051 & -5.25 & \\
\hline 13 & 4 & 10 & 14 & 4 & 11 & 53487.550 & -0.084 & -5.24 & \\
\hline 13 & 5 & 8 & 14 & 5 & 9 & 53467.320 & $\odot .099$ & -4.13 & \\
\hline 13 & 5 & 9 & 14 & 5 & 10 & 53467.320 & 0.129 & -4.13 & \\
\hline 13 & 6 & 7 & 14 & 6 & 8 & 53456.320 & -0.455 & -2.79 & \\
\hline 13 & 6 & 8 & 14 & 6 & 9 & 53456.320 & -0.455 & -2.79 & \\
\hline 14 & 1 & 13 & 15 & 0 & 15 & 36968.990 & -0.157 & -0.39 & \\
\hline 14 & 2 & 12 & 15 & 1 & 14 & 35477.650 & -0.054 & -11.61 & \\
\hline 14 & 2 & 12 & 15 & 2 & 13 & 57986.900 & $\odot .454$ & -9.51 & \\
\hline 14 & 2 & 13 & 15 & 2 & 14 & 57063.050 & 0.183 & -7.74 & \\
\hline 14 & 3 & 11 & 15 & 3 & 12 & 57429.790 & 0.094 & -7.95 & \\
\hline 14 & 3 & 12 & 15 & 3 & 13 & 57335.670 & -0.170 & -7.66 & \\
\hline 14 & 4 & 10 & 15 & 4 & 11 & $57319.30 \odot$ & -0.003 & -6.78 & \\
\hline 14 & 4 & 11 & 15 & 4 & 12 & 57316.110 & $\odot .078$ & -6.76 & \\
\hline 14 & 5 & 9 & 15 & 5 & 10 & 57291.180 & 0.056 & -5.56 & \\
\hline 14 & 5 & 10 & 15 & 5 & 11 & 57291.180 & 0.113 & -5.56 & \\
\hline 14 & 6 & 8 & 15 & 6 & 9 & 57278.220 & 0.169 & -4.12 & \\
\hline 14 & 6 & 9 & 15 & 6 & 10 & 57278.220 & 0.169 & -4.12 & \\
\hline 15 & 2 & 13 & 16 & 1 & 15 & 39370.700 & 0.382 & -13.19 & \\
\hline 16 & 1 & 15 & 16 & 2 & 15 & 17252.050 & 0.074 & 9.87 & \\
\hline 15 & 4 & 11 & 16 & 4 & 12 & 61150.940 & -0.108 & -8.56 & \\
\hline 15 & 4 & 12 & 16 & 4 & 13 & 61145.640 & -0.245 & -8.53 & \\
\hline 15 & 5 & 10 & 16 & 5 & 11 & 61115.970 & -0.062 & -7.24 & \\
\hline 15 & 5 & 11 & 16 & 5 & 12 & 61115.970 & $\odot .041$ & -7.24 & \\
\hline 15 & 6 & 9 & 16 & 6 & 10 & 61099.820 & -0.114 & -5.69 & \\
\hline 15 & 6 & 10 & 16 & 6 & 11 & 61099.820 & -0.113 & -5.69 & \\
\hline 16 & 1 & 15 & 17 & 0 & 17 & 40618.400 & -0.122 & 1.70 & -0.01 \\
\hline 16 & 2 & 14 & 17 & 1 & 16 & 43135.300 & 0.793 & -14.64 & \\
\hline 17 & 1 & 16 & 18 & $\odot$ & 18 & 42211.400 & $\odot .137$ & 3.23 & -0.01 \\
\hline 18 & 1 & 17 & 18 & 2 & 17 & 15093.520 & 0.118 & 13.67 & -0.01 \\
\hline 20 & 1 & 19 & 20 & 2 & 19 & 12891.100 & 0.095 & 17.85 & -0.01 \\
\hline 20 & 2 & 18 & 20 & 3 & 18 & 37084.400 & $\odot .090$ & 22.96 & -0.01 \\
\hline 21 & 3 & 18 & 21 & 4 & 18 & 60722.990 & -0.068 & 16.77 & \\
\hline 23 & 6 & 17 & 22 & 7 & 15 & 28571.200 & -0.083 & 24.62 & \\
\hline 23 & 6 & 18 & 22 & 7 & 16 & 28571.200 & -0.260 & 24.61 & \\
\hline 25 & 2 & 23 & 25 & 3 & 23 & 30188.910 & -0.029 & 49.63 & -0.04 \\
\hline 25 & 3 & 22 & 25 & 4 & 22 & 58064.140 & -0.062 & 37.76 & $-\odot .02$ \\
\hline 26 & 2 & 24 & 26 & 3 & 24 & 28633.440 & 0.016 & 56.18 & -0.04 \\
\hline 27 & 2 & 25 & 27 & 3 & 25 & 27040.510 & -0.121 & 63.01 & -0.05 \\
\hline 28 & 7 & 21 & 27 & 8 & 19 & 27348.080 & $\odot .233$ & 51.16 & \\
\hline
\end{tabular}




\begin{tabular}{|c|c|c|c|c|c|c|c|c|}
\hline 28 & 2 & 26 & 28 & 326 & 25420.750 & -0.140 & 70.03 & -0.07 \\
\hline 28 & 3 & 25 & 28 & 425 & 54892.620 & $-\odot .017$ & 64.11 & -0.05 \\
\hline 29 & 2 & 27 & 29 & 327 & 23785.110 & 0.035 & 77.13 & $-\odot .08$ \\
\hline 30 & 2 & 28 & 30 & 328 & 22144.600 & $\odot .030$ & 84.18 & $-\odot . \odot 9$ \\
\hline 31 & 2 & 29 & 31 & 329 & 20511.190 & 0.006 & 91.01 & -0.11 \\
\hline 32 & 2 & 30 & 32 & 330 & 18897.300 & 0.291 & 97.47 & -0.13 \\
\hline 33 & 2 & 31 & 33 & 331 & 17314.250 & $\odot .046$ & 103.36 & -0.14 \\
\hline 35 & 2 & 33 & 35 & 333 & 14289.990 & 0.030 & 112.73 & -0.18 \\
\hline 36 & 2 & 34 & 36 & 334 & 12870.540 & 0.130 & 115.90 & -0.19 \\
\hline 37 & 3 & 34 & 37 & 434 & 39562.270 & $\odot .152$ & 198.93 & -0.33 \\
\hline 39 & 3 & 36 & 39 & 436 & 35381.680 & 0.063 & 235.00 & -0.44 \\
\hline 40 & 3 & 37 & 40 & 437 & 33254.410 & 0.060 & 252.68 & -0.51 \\
\hline 41 & 3 & 38 & 41 & 438 & 31119.750 & $-\odot . \odot 92$ & 269.75 & -0.57 \\
\hline 42 & 3 & 39 & 42 & 439 & 28991.530 & -0.041 & 285.85 & -0.64 \\
\hline 43 & 3 & 40 & 43 & 440 & 26883.110 & -0.105 & 300.64 & -0.71 \\
\hline 44 & 3 & 41 & 44 & 441 & 24808.340 & $-\odot .156$ & 313.75 & -0.78 \\
\hline 48 & 3 & 45 & 48 & 445 & 17108.490 & 0.004 & 342.70 & -1.03 \\
\hline 48 & 4 & 44 & 48 & 544 & 51206.110 & $-\odot .046$ & 507.48 & -1.44 \\
\hline 51 & 3 & 48 & 51 & 448 & 12269.010 & -0.011 & 334.15 & -1.15 \\
\hline 55 & 4 & 51 & 55 & 551 & 33306.080 & 0.119 & 745.06 & -2.92 \\
\hline 57 & 4 & 53 & 57 & 553 & 28360.740 & -0.030 & 783.60 & -3.3 \\
\hline 58 & 4 & 54 & 58 & 554 & 25990.710 & -0.100 & 795.03 & -3.5 \\
\hline 60 & 4 & 56 & 60 & 556 & 21522.690 & 0.074 & 800.16 & -3.8 \\
\hline
\end{tabular}

RMS $=0.1467 \quad$ GRAMDET.$=0.68 \mathrm{E}-12$

ROTATIONAL CONSTANTS (MHZ) :

$\begin{array}{rrrr} & 10878.235115 & 1984.768190 & 1831.614416 \\ +- & 0.016517 & 0.001907 & 0.001813\end{array}$

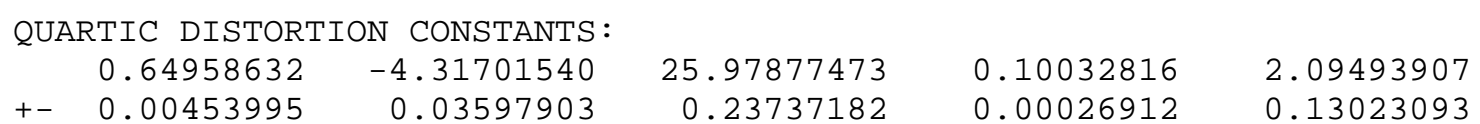

\begin{tabular}{|c|c|c|c|c|}
\hline \multicolumn{5}{|c|}{ SEXTIC DIST } \\
\hline & $\odot . ๑ \odot \odot \odot \odot \odot \odot \odot$ & $\odot . \odot \odot \odot \odot \odot \odot \odot \odot$ & $\odot . ๑ \odot \odot \odot \odot \odot \odot \odot$ & $\odot .0000000 \odot$ \\
\hline+- & ๑. . 00000000 & ๑. . 00000000 & ๑. . 00000000 & ๑. . 00000000 \\
\hline & ๑. $00 \odot 16518$ & 0.00000 & $\odot .000 \odot \odot \odot \odot \odot$ & \\
\hline+- & $\odot .00003493$ & $\odot .00 \odot \odot \odot \odot \odot \odot$ & $\odot .0 \odot \odot \odot \odot \odot \odot \odot$ & \\
\hline
\end{tabular}

\section{SIGNIFICANT DIGITS AND CORRELATION MATRIX:}

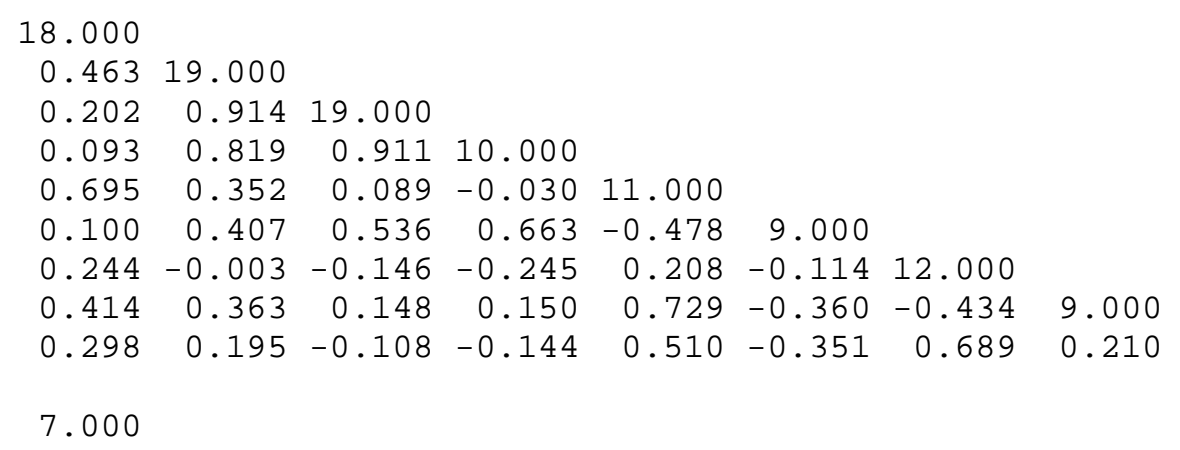




\section{DERIVED CONSTANTS:}

KAPPA $=-0.966141$

MOMENTS OF INERTIA AND DEFECT $(U * A * * 2)$ :

$46.45781643254 .62875344275 .92000018-25.16656969$

$\begin{array}{lllll}+- & 0.00007054 & 0.00024467 & 0.00027319 & 0.00015939\end{array}$

STANDARD DISTORTION CONSTANTS (KHZ):

$\begin{array}{lll}-89.245383 & -3.400971 & -1.795720 \\ -2.745383 & -2.598345 & 14.816754\end{array}$


Table 2S: Microwave Spectrum and Spectroscopic Constants of Conformer III of Cyclopropylmethylphosphine in Vibrationally Excited State IIIa

TOTAL NUMBER OF ACCEPTED TRANSITIONS: 24

TRANSITION

$(\mathrm{J}, \mathrm{K}-1, \mathrm{~K}+1-->\mathrm{J}$,

$\begin{array}{rrrrrr}8 & 3 & 5 & 9 & 3 & 6 \\ 8 & 3 & 6 & 9 & 3 & 7 \\ 12 & 2 & 10 & 13 & 2 & 11 \\ 12 & 2 & 11 & 13 & 2 & 12 \\ 12 & 4 & 8 & 13 & 4 & 9 \\ 12 & 5 & 7 & 13 & 5 & 8 \\ 12 & 5 & 8 & 13 & 5 & 9 \\ 12 & 6 & 6 & 13 & 6 & 7 \\ 12 & 6 & 7 & 13 & 6 & 8 \\ 13 & 4 & 9 & 14 & 4 & 10 \\ 13 & 4 & 10 & 14 & 4 & 11 \\ 14 & 3 & 11 & 15 & 3 & 12 \\ 14 & 4 & 10 & 15 & 4 & 11 \\ 14 & 4 & 11 & 15 & 4 & 12 \\ 14 & 5 & 9 & 15 & 5 & 10 \\ 14 & 5 & 10 & 15 & 5 & 11 \\ 14 & 6 & 8 & 15 & 6 & 9 \\ 14 & 6 & 9 & 15 & 6 & 10 \\ 15 & 4 & 11 & 16 & 4 & 12 \\ 15 & 4 & 12 & 16 & 4 & 13 \\ 15 & 5 & 10 & 16 & 5 & 11 \\ 15 & 5 & 11 & 16 & 5 & 12 \\ 15 & 6 & 9 & 16 & 6 & 10 \\ 15 & 6 & 10 & 16 & 6 & 11\end{array}$

OBS . FREQ. $0 .-\mathrm{C}$.

FREQ .

34348.950

34342.000

50081.650

49449.600

49613.070

49595.880

49595.880

49588.350

49588.350

53437.230

53435.000

57371.110

57262.810

57259.640

57235.170

57235.170

57222.540

61090.490

61085.410

61056.200

61056.200

$61040.620-0.076$

$61040.620-0.075$

RMS $=0.2214 \quad$ GRAMDET.$=0.32 \mathrm{E}-01$

ROTATIONAL CONSTANTS (MHZ):

$$
\begin{array}{rrrr}
10878.000000 & 1982.243364 & 1830.427436 \\
+- & 0.000000 & 0.016751 & 0.019017
\end{array}
$$

\begin{tabular}{|c|c|c|c|}
\hline & & 29 & $\odot \odot$ \\
\hline 00 & $\odot . \odot \odot \odot \odot \odot \odot \odot \odot$ & $\odot . \odot \odot \odot \odot \odot \odot \odot \odot$ & $\odot . \odot \odot \odot \odot \odot \odot \odot \odot$ \\
\hline
\end{tabular}

QUARTIC DISTORTION CONSTANTS:

SIGNIFICANT DIGITS AND CORRELATION MATRIX:

14.000

$-0.98414 .000$ 


\section{DERIVED CONSTANTS:}

KAPPA $=-0.966441$

MOMENTS OF INERTIA AND DEFECT $\left(U^{*} A^{* *} 2\right)$ :

$46.45882056254 .95307948 \quad 276.09892644 \quad-25.31297359$

$\begin{array}{llll}+- & 0.00000000 & 0.00215446 & 0.00286849\end{array}$

STANDARD DISTORTION CONSTANTS (KHZ):

$\begin{array}{rrr}-101.600000 & -3.200000 & -1.600000 \\ -3.200000 & -2.400000 & 16.000000\end{array}$


Table 3S: Microwave Spectrum and Spectroscopic Constants of Conformer III of Cyclopropylmethylphosphine in Vibrationally Excited State IIIb

TOTAL NUMBER OF ACCEPTED TRANSITIONS: 18

TRANSITION OBS.FREQ. O.-C. $\begin{gathered}\text { DISTORTION } \\ \text { TOTAL }\end{gathered} \underset{\text { FIXND }}{\text { CONIBUTION }}$

\begin{tabular}{|c|c|c|c|c|c|c|c|c|c|}
\hline 6 & 3 & 3 & 7 & 3 & 4 & 26745.060 & $\odot .049$ & $-\odot .27$ & -0.27 \\
\hline 6 & 3 & 4 & 7 & 3 & 5 & 26743.160 & 0.111 & $-\odot .27$ & -0.27 \\
\hline 8 & 3 & 5 & 9 & 3 & 6 & 34400.190 & $\odot .347$ & -1.05 & -1.05 \\
\hline 8 & 3 & 6 & 9 & 3 & 7 & 34392.770 & $\odot .099$ & -1.04 & -1.04 \\
\hline 13 & 4 & 9 & 14 & 4 & 10 & 53517.010 & $\odot .290$ & -4.66 & -4.66 \\
\hline 13 & 4 & 10 & 14 & 4 & 11 & 53514.750 & 0.019 & -4.65 & -4.65 \\
\hline 13 & 5 & 8 & 14 & 5 & 9 & 53494.420 & $\odot .0 \odot 4$ & -3.52 & -3.52 \\
\hline 13 & 5 & 9 & 14 & 5 & 10 & 53494.420 & 0.034 & -3.52 & -3.52 \\
\hline 14 & 4 & 10 & 15 & 4 & 11 & 57348.430 & $\odot .064$ & -6.06 & -6.06 \\
\hline 14 & 4 & 11 & 15 & 4 & 12 & $57345.10 \odot$ & $-\odot .019$ & -6.05 & -6.05 \\
\hline 14 & 6 & 8 & 15 & 6 & 9 & 57307.580 & 0.246 & -3.36 & -3.36 \\
\hline 14 & 6 & 9 & 15 & 6 & 10 & 57307.580 & 0.246 & -3.36 & -3.36 \\
\hline 15 & 4 & 11 & 16 & 4 & 12 & 61182.150 & $\odot .049$ & -7.69 & -7.69 \\
\hline 15 & 4 & 12 & 16 & 4 & 13 & 61176.930 & $-\odot . \odot 46$ & -7.67 & -7.67 \\
\hline 15 & 5 & 10 & 16 & 5 & 11 & 61146.810 & $-\odot .445$ & -6.35 & -6.35 \\
\hline 15 & 5 & 11 & 16 & 5 & 12 & 61146.810 & $-\odot .342$ & -6.35 & -6.35 \\
\hline 15 & 6 & 9 & 16 & 6 & 10 & 61131.080 & $-\odot .172$ & -4.78 & -4.78 \\
\hline 15 & 6 & 10 & 16 & 6 & 11 & 61131.080 & $-\odot .171$ & -4.78 & -4.78 \\
\hline
\end{tabular}

RMS $=0.2143 \quad$ GRAMDET.$=0.11 \mathrm{E}-02$

ROTATIONAL CONSTANTS (MHZ):

$\begin{array}{rrrr} & 10878.0000 \odot \odot & 1985.573566 & 1832.719451 \\ +- & 0.0000 \odot \odot & 0.104341 & 0.115591\end{array}$

QUARTIC DISTORTION CONSTANTS:

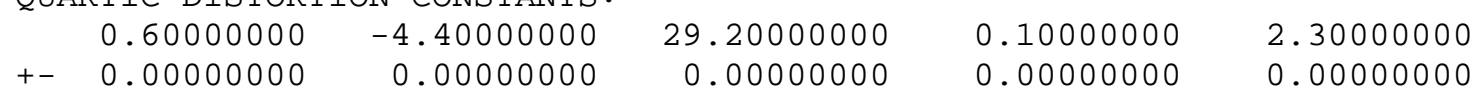

SIGNIFICANT DIGITS AND CORRELATION MATRIX:

14.000

$-0.99914 .000$

DERIVED CONSTANTS:

KAPPA $=-0.966202$

MOMENTS OF INERTIA AND DEFECT $\left(U^{*} A^{* *} 2\right)$ :

$46.45882056254 .52547246275 .75363468-25.23065833$

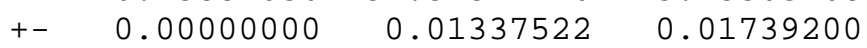


STANDARD DISTORTION CONSTANTS (KHZ):

$\begin{array}{rrr}-101.600000 & -3.2000000 & -1.600000 \\ -3.200000 & -2.400000 & 16.000000\end{array}$


Table 4S: Microwave Spectrum and Spectroscopic Constants of the Ground Vibrational State of Cyclopropylmethylphosphine, Conformer IV

TOTAL NUMBER OF ACCEPTED TRANSITIONS: 114

TRANSITION
OBS. FREQ. O. - C.

FREQ .

DELTA
$15276.420-0.052$

$14714.350-0.003$

$15013.600-0.178$

$14997.570 \quad 0.085$

$15002.060-0.120$

$15002.060-0.049$

$22440.800-0.091$

$22905.910-0.078$

$22063.670 \quad 0.237$

$22547.250-0.111$

$22490.480-0.061$

$22503.800-0.088$

$22503.800-0.086$

$22502.720-0.050$

$22502.720-0.050$

$26156.610 \quad 0.055$

$26716.660-0.232$

$25734.580-0.097$

$26324.980-0.248$

$26234.510-0.145$

$26261.670-0.190$

$26260.190-0.182$

$26255.690-0.305$

$26255.690-0.297$

$29861.700 \quad 0.350$

$30524.640 \quad 0.133$

$29403.110-0.205$

$30111.780-0.101$

29977.160

0.377

30018.040

0.019

$30014.890-0.159$

$30008.690-0.088$

$30008.690-0.065$

$30005.570-0.185$

$30005.570-0.185$

$30004.310-0.194$

$30004.310-0.194$

$33554.380 \quad 0.186$

$34328.210-0.034$

33069.140

33717.110

0.053

33776.710

0.464

33771.400

0.495

33762.730

0.626

0.391

$\odot .447$
$-0.13$

$-0.14$

$-0.08$

$-0.04$

$-0.03$

0.10

0.10

$-0.44$

$-0.50$

$-0.33$

$-0.32$

$-0.30$

0.16

0.16

0.52

0.52

$-0.69$

$-0.79$

$-0.55$

$-0.58$

$-0.53$

$-0.32$

$-0.32$

$-1.01$

$-1.19$

$-0.84$

$-0.94$

$-0.86$

$-0.63$

$-0.62$

$-0.26$

$-0.26$

0.21

0.21

0.78

0.78

$-1.42$

$-1.69$

$-1.22$

$-1.28$

$-1.04$

$-1.03$

$-0.62$

$-0.62$
0.08

$-0.14$

0.20

$-0.05$

$-0.03$

$\odot .04$

๑. 02

$-0.07$

0.09

-0.01
0.01

$-0.01$

$-0.01$

$-0.10$

0.14

$-0.03$

$\odot .02$

0.03

$-0.01$

$-0.01$

$-0.01$

$-0.01$

0.13

$-0.19$

0.28

0.04

$-0.01$ 


\begin{tabular}{|c|c|c|c|c|c|c|c|c|c|}
\hline 8 & 5 & 3 & 9 & 5 & 4 & 33758.390 & 0.508 & $-\odot .09$ & -0.01 \\
\hline 8 & 5 & 4 & 9 & 5 & 5 & 33758.390 & 0.508 & $-\odot .09$ & -0.01 \\
\hline 8 & 6 & 2 & 9 & 6 & 3 & 33756.460 & 0.529 & 0.56 & -0.01 \\
\hline 8 & 6 & 3 & 9 & 6 & 4 & 33756.460 & $\odot .529$ & $\odot .56$ & $-\odot .01$ \\
\hline 9 & $\odot$ & 9 & 10 & $0=$ & 10 & 37234.230 & $-\odot .088$ & -1.92 & 0.21 \\
\hline 9 & 1 & 8 & 10 & 1 & 9 & 38127.540 & $\odot .076$ & -2.31 & -0.24 \\
\hline 9 & 1 & 9 & 10 & $1:$ & 10 & 36731.750 & $-\odot .023$ & -1.69 & 0.38 \\
\hline 9 & 2 & 7 & 10 & 2 & 8 & 37714.300 & $-\odot .046$ & -2.03 & -0.14 \\
\hline 9 & 2 & 8 & 10 & 2 & 9 & 37453.740 & -0.228 & -1.81 & 0.07 \\
\hline 9 & 3 & 6 & 10 & 3 & 7 & 37536.590 & -0.260 & -1.57 & $-\odot .01$ \\
\hline 9 & 3 & 7 & 10 & 3 & 8 & 37527.510 & $-\odot . \odot 29$ & -1.55 & \\
\hline 9 & 4 & 5 & 10 & 4 & 6 & 37516.510 & -0.271 & -1.09 & \\
\hline 9 & 4 & 6 & 10 & 4 & 7 & 37516.510 & $-\odot .149$ & -1.09 & \\
\hline 9 & 5 & 4 & 10 & 5 & 5 & 37510.370 & -0.136 & $-\odot .5 \odot$ & \\
\hline 9 & 5 & 5 & 10 & 5 & 6 & 37510.370 & $-\odot .136$ & $-\odot .5 \odot$ & \\
\hline 9 & 6 & 3 & 10 & 6 & 4 & 37507.490 & -0.172 & 0.21 & -0.01 \\
\hline 9 & 6 & 4 & 10 & 6 & 5 & 37507.490 & $-\odot .172$ & 0.21 & $-\odot .01$ \\
\hline 10 & 2 & 8 & 11 & 2 & 9 & 41530.910 & 0.220 & -2.79 & -0.21 \\
\hline 10 & 4 & 6 & 11 & 4 & 7 & 41272.390 & 0.177 & -1.70 & \\
\hline 10 & 4 & 7 & 11 & 4 & 8 & 41271.610 & -0.360 & -1.70 & \\
\hline 10 & 5 & 5 & 11 & 5 & 6 & 41263.310 & -0.373 & -1.05 & \\
\hline 10 & 5 & 6 & 11 & 5 & 7 & 41263.310 & -0.371 & -1.05 & \\
\hline 10 & 6 & 4 & 11 & 6 & 5 & 41259.720 & $-\odot .011$ & -0.26 & -0.01 \\
\hline 10 & 6 & 5 & 11 & 6 & 6 & 41259.720 & -0.011 & -0.26 & -0.01 \\
\hline 11 & 2 & 9 & 12 & $2=$ & 10 & 45356.560 & -0.183 & -3.72 & -0.31 \\
\hline 11 & 21 & 10 & 12 & 2 & 11 & 44919.940 & 0.044 & -3.24 & 0.14 \\
\hline 11 & 3 & 8 & 12 & 3 & 9 & 45067.470 & 0.126 & -3.04 & $-\odot . \odot 4$ \\
\hline 11 & 3 & 9 & 12 & 3 & 10 & 45044.240 & $\odot .298$ & -3.00 & \\
\hline 11 & 5 & 6 & 12 & 5 & 7 & 45017.490 & 0.023 & -1.73 & \\
\hline 11 & 5 & 7 & 12 & 5 & 8 & 45017.490 & $\odot .027$ & -1.73 & \\
\hline 12 & 21 & 10 & 13 & 2 & 11 & 49191.260 & -0.375 & -4.84 & -0.44 \\
\hline 12 & 21 & 11 & 13 & $2=$ & 12 & 48647.820 & -0.155 & -4.17 & 0.19 \\
\hline 12 & 3 & 9 & 13 & $3=$ & 10 & 48838.340 & 0.041 & -4.02 & $-\odot .07$ \\
\hline 12 & 31 & 10 & 13 & $3=$ & 11 & 48803.800 & $\odot .458$ & -3.95 & $-\odot .01$ \\
\hline 12 & 4 & 8 & 13 & 4 & 9 & 48786.870 & 0.352 & -3.35 & -0.01 \\
\hline 12 & 4 & 9 & 13 & $4=$ & 10 & 48785.630 & $-\odot . \odot 80$ & -3.35 & \\
\hline 12 & 5 & 7 & 13 & 5 & 8 & $48772 . \odot \odot \odot$ & $\odot .085$ & -2.57 & \\
\hline 12 & 5 & 8 & 13 & 5 & 9 & $48772 . \odot \odot \odot$ & $\odot .094$ & -2.57 & \\
\hline 12 & 6 & 6 & 13 & 6 & 7 & 48765.120 & $\odot .102$ & -1.63 & \\
\hline 12 & 6 & 7 & 13 & 6 & 8 & 48765.120 & 0.102 & -1.63 & \\
\hline 13 & 21 & 11 & 14 & 2 & 12 & 53034.140 & 0.100 & -6.15 & -0.60 \\
\hline 13 & 21 & 12 & 14 & $2=$ & 13 & 52372.420 & $-\odot .036$ & -5.24 & 0.26 \\
\hline 13 & 31 & 10 & 14 & 3 & 11 & 52613.710 & -0.178 & -5.18 & -0.11 \\
\hline 13 & 31 & 11 & 14 & $3=$ & 12 & 52563.100 & $-\odot .206$ & -5.07 & $-\odot .01$ \\
\hline 13 & 4 & 9 & 14 & $4=$ & 10 & 52545.280 & -0.370 & -4.43 & -0.01 \\
\hline 13 & 41 & 10 & 14 & $4=$ & 11 & 52544.480 & 0.202 & -4.42 & $-\odot .01$ \\
\hline 13 & 5 & 8 & 14 & 5 & 9 & 52526.960 & -0.124 & -3.58 & \\
\hline 13 & 5 & 9 & 14 & $5:$ & 10 & 52526.960 & -0.105 & -3.58 & \\
\hline 13 & 6 & 7 & 14 & 6 & 8 & 52518.130 & -0.175 & -2.57 & \\
\hline 13 & 6 & 8 & 14 & 6 & 9 & 52518.130 & $-\odot .174$ & -2.57 & \\
\hline 14 & 21 & 12 & 15 & $2=$ & 13 & 56882.450 & 0.202 & -7.68 & -0.78 \\
\hline 14 & 21 & 13 & 15 & $2=$ & 14 & 56092.910 & $-\odot .188$ & -6.48 & 0.34 \\
\hline 14 & 31 & 11 & 15 & $3=$ & 12 & 56394.790 & $-\odot .0 \odot 6$ & -6.54 & -0.18 \\
\hline 14 & 31 & 12 & 15 & $3=$ & 13 & 56323.570 & $-\odot .033$ & -6.36 & $-\odot .01$ \\
\hline 14 & 41 & 10 & 15 & $4=$ & 11 & 56306.070 & $-\odot .226$ & -5.69 & -0.03 \\
\hline 14 & 41 & 11 & 15 & $4=$ & 12 & 56303.990 & $-\odot .066$ & -5.68 & -0.02 \\
\hline 14 & 5 & 9 & 15 & 5 & 10 & 56283.050 & $\odot .019$ & -4.77 & \\
\hline
\end{tabular}




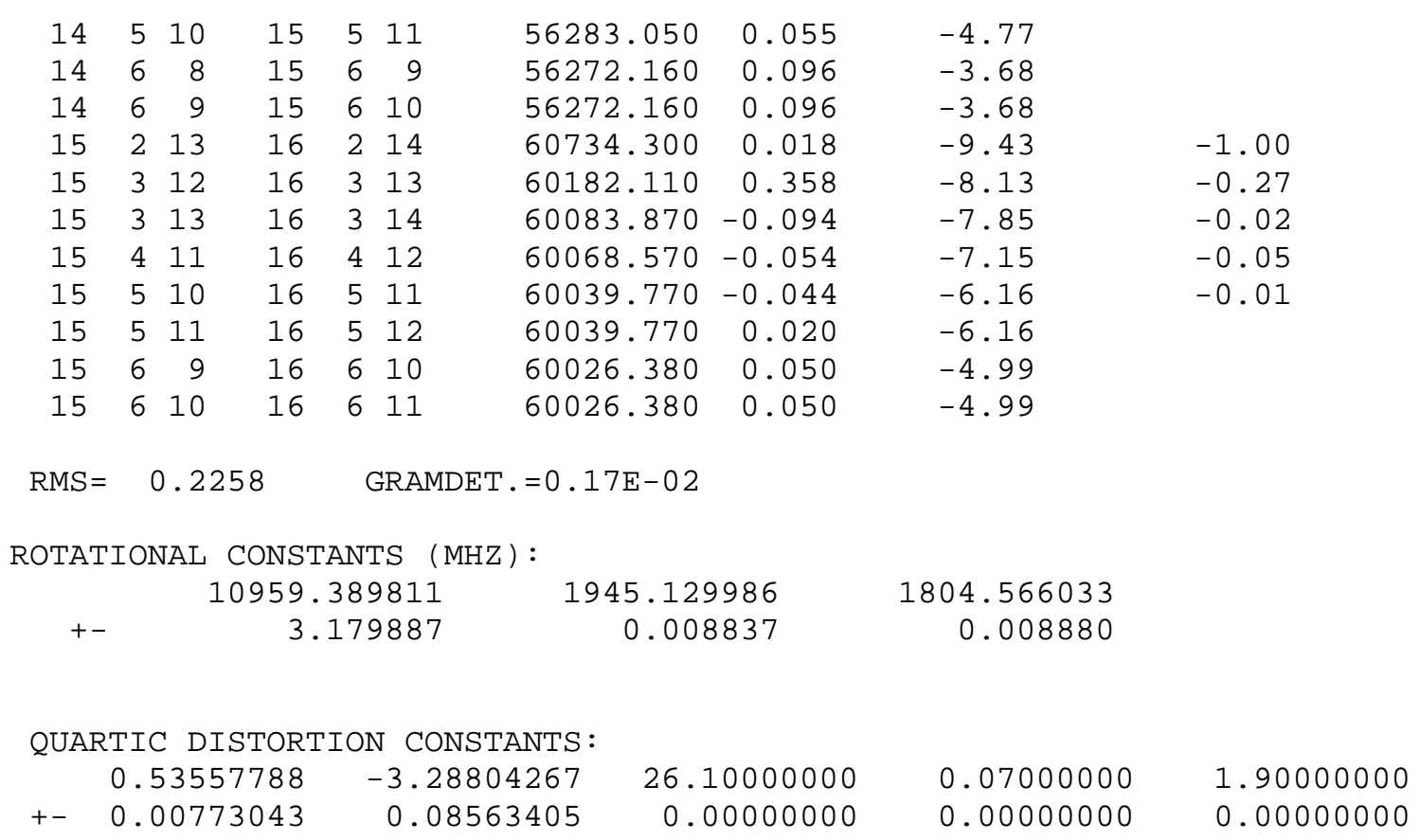

SIGNIFICANT DIGITS AND CORRELATION MATRIX:

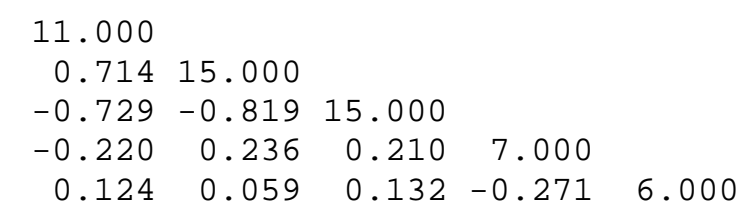

DERIVED CONSTANTS:

KAPPA $=-\odot .969292$

MOMENTS OF INERTIA AND DEFECT $\left(U^{*} A * * 2\right)$ :

$\begin{array}{lllll}46.11379454 & 259.81762327 & 280.05572577 & -25.87569204\end{array}$

+ $\quad 0.01338000 \quad 0.00118040 \quad 0.00137815 \quad 0.01531092$

STANDARD DISTORTION CONSTANTS (KHZ):

$\begin{array}{lll}-93.390141 & -2.702312 & -1.582312 \\ -3.446226 & -2.142312 & 12.313774\end{array}$


Table 5S: Microwave Spectrum and Spectroscopic Constants of Conformer IV of Cyclopropylmethylphosphine in Vibrationally Excited State IVa

TOTAL NUMBER OF ACCEPTED TRANSITIONS: 83

TRANSITION
OBS. FREQ. O. - C.

FREQ .

DELTA
$22462.410-0.101$

$22462.410-0.099$

$22461.310-0.124$

$22461.310-0.124$

$26662.300-0.151$

$25693.900-0.201$

$26212.860-0.501$

$26211.790 \quad-0.144$

$26207.340-0.337$

$26207.340-0.329$

$26205.430-0.387$

$26205.430-0.387$

$29810.010-0.041$

$30462.280-0.260$

$29357.000-0.142$

$30053.660-0.134$

$29922.780 \quad 0.417$

$29962.740 \quad 0.284$

29960.080

29953.900

0.475

29953.900

29950.860

0.402

0.424

0.287

29950.860

0.287

29949.710

$\odot .335$

29949.710

0.335

$33497.420-0.099$

34258.920

$\odot .058$

33017.500

0.112

0.361

33708.540

0.242

33700.360

0.287

33700.360

0.340

33696.190

0.434

$33696.190 \quad 0.434$

37172.580

38050.900

0.015

36674.660

0.102

$37639.750-0.073$

$37386.360-0.032$

$37466.730-0.213$

$37458.010 \quad 0.000$

$37447.300-0.202$

$37447.300-0.087$

$37441.270-0.150$

$37441.270-0.149$
$-0.11$

0.17

0.17

0.52

0.52

$-0.79$

$-0.55$

$-0.32$

$-0.32$

0.42

0.42

$-1.01$

$-1.18$

$-0.84$

$-0.94$

$-0.85$

$-0.62$

$-0.62$

$-0.25$

$-0.25$

0.22

๑. 22

0.80

0.80

$-1.42$

$-1.68$

$-1.21$

$-1.03$

$-1.02$

$-0.61$

$-0.61$

- 0.08

$-0.08$

$-1.92$

$-2.30$

$-1.68$

$-2.02$

$-1.80$

$-1.55$

$-1.54$

$-1.08$

$-1.08$

$-0.49$

$-0.49$
0.35

0.63

0.63

0.98

$\odot .98$

$-0.06$

$\odot .19$

0.41

0.41

1.15

1.15

0.08

$-0.09$

0.25

0.16

0.24

0.47

0.47

0.84

0.84

1. 31

1.31

1.89

1.89

0.14

$-0.13$

0.34

0.53

$\odot .53$

$\odot .95$

0.95

1.48

1.48

๑. 22

$-0.17$

0.45

0.12

0.33

0.58

0.59

1.05

1.05

1.65

1.65 


\begin{tabular}{|c|c|c|c|c|c|c|c|c|}
\hline 11 & 2 & 12 & 2 & 10 & 45264.760 & -0.225 & -3.70 & -0.02 \\
\hline 11 & 4 & 12 & 4 & 8 & 44944.970 & -0.378 & -2.43 & 1.26 \\
\hline 11 & 4 & 12 & 4 & 9 & 44944.970 & 0.053 & -2.42 & 1.26 \\
\hline 11 & 5 & 12 & 5 & 7 & 44934.770 & $\odot .367$ & -1.71 & 1.98 \\
\hline 11 & 5 & 12 & 5 & 8 & 44934.770 & $\odot .371$ & -1.71 & 1.98 \\
\hline 11 & 6 & 12 & 6 & 6 & 44928.970 & -0.310 & -0.84 & 2.85 \\
\hline 11 & 6 & 12 & 6 & 7 & 44928.970 & -0.310 & -0.84 & 2.85 \\
\hline 12 & 210 & 13 & 2 & 11 & 49090.880 & -0.197 & -4.81 & -0.13 \\
\hline 12 & 4 & 13 & 4 & 9 & 48695.970 & -0.026 & -3.33 & 1.36 \\
\hline 12 & 4 & 13 & 4 & 10 & 48694.920 & -0.311 & -3.32 & 1.36 \\
\hline 13 & 211 & 14 & 2 & 12 & 52924.670 & 0.019 & -6.12 & -0.27 \\
\hline 13 & 212 & 14 & 2 & 13 & 52279.380 & -0.126 & -5.22 & $\odot .63$ \\
\hline 13 & 311 & 14 & 3 & 12 & 52465.360 & $-\odot .074$ & -5.04 & $\odot .82$ \\
\hline 13 & 4 & 14 & 4 & 10 & 52448.180 & 0.215 & -4.40 & 1.46 \\
\hline 13 & 410 & 14 & 4 & 11 & 52446.180 & $-\odot .486$ & -4.39 & 1.46 \\
\hline 13 & 5 & 14 & 5 & 9 & 52429.740 & -0.214 & -3.55 & 2.31 \\
\hline 13 & 5 & 14 & 5 & 10 & 52429.740 & $-\odot .197$ & -3.55 & 2.31 \\
\hline 13 & 6 & 14 & 6 & 8 & 52421.360 & $-\odot .089$ & -2.53 & 3.32 \\
\hline 13 & 6 & 14 & 6 & 9 & 52421.360 & $-\odot .088$ & -2.53 & 3.32 \\
\hline 14 & 212 & 15 & 2 & 13 & 56764.330 & 0.255 & -7.64 & -0.44 \\
\hline 14 & 213 & 15 & 2 & 14 & 55994.090 & $\odot .055$ & -6.45 & 0.75 \\
\hline 14 & 311 & 15 & 3 & 12 & 56287.080 & $\odot .082$ & -6.51 & $\odot .69$ \\
\hline 14 & 312 & 15 & 3 & 13 & 56218.910 & $\odot .257$ & -6.33 & $\odot .87$ \\
\hline 14 & 410 & 15 & 4 & 11 & 56201.310 & $-\odot .087$ & -5.65 & 1.55 \\
\hline 14 & 411 & 15 & 4 & 12 & 56199.360 & $\odot .084$ & -5.64 & 1.56 \\
\hline 14 & 5 & 15 & 5 & 10 & 56178.610 & -0.221 & -4.73 & 2.47 \\
\hline 14 & 510 & 15 & 5 & 11 & 56178.610 & -0.187 & -4.73 & 2.47 \\
\hline 14 & 68 & 15 & 6 & 9 & 56168.090 & -0.110 & -3.64 & 3.56 \\
\hline 14 & 6 & 15 & 6 & 10 & 56168.090 & -0.110 & -3.64 & 3.56 \\
\hline 15 & 312 & 16 & 3 & 13 & $6 \odot \odot 65.80 \odot$ & -0.054 & -8.08 & $\odot .66$ \\
\hline 15 & 313 & 16 & 3 & 14 & 59972.010 & $\odot .053$ & -7.82 & $\odot .92$ \\
\hline 15 & 411 & 16 & 4 & 12 & 59956.740 & 0.287 & -7.11 & 1.63 \\
\hline 15 & 412 & 16 & 4 & 13 & 59953.480 & 0.375 & -7.09 & 1.65 \\
\hline 15 & 510 & 16 & 5 & 11 & 59928.520 & $\odot .0 \odot 4$ & -6.11 & 2.63 \\
\hline 15 & 511 & 16 & 5 & 12 & 59928.520 & 0.065 & -6.11 & 2.63 \\
\hline 15 & 6 & 16 & 6 & 10 & 59915.500 & 0.059 & -4.94 & 3.80 \\
\hline 15 & 610 & 16 & 6 & 11 & 59915.500 & 0.060 & -4.94 & 3.80 \\
\hline
\end{tabular}

RMS $=0.2489 \quad$ GRAMDET.$=0.60 \mathrm{E}-02$

ROTATIONAL CONSTANTS (MHZ):

$\begin{array}{rrrr} & 10950.165888 & 1940.699591 & 1802.124943 \\ +- & 3.889052 & 0.010607 & 0.010651\end{array}$

QUARTIC DISTORTION CONSTANTS:

\begin{tabular}{|c|c|c|c|c|}
\hline 0.5334465 & - 3.30000000 & 106 & & \\
\hline & ๑.००००००० & ๑.๑०९०९००९ & & \\
\hline
\end{tabular}

\section{SIGNIFICANT DIGITS AND CORRELATION MATRIX:}

$$
\begin{array}{rrrr}
11.000 & & & \\
0.691 & 15.000 & & \\
-0.723 & -0.822 & 15.00 \odot & \\
-0.177 & 0.265 & 0.279 & 6.00 \odot
\end{array}
$$


DERIVED CONSTANTS:

KAPPA $=-0.969704$

MOMENTS OF INERTIA AND DEFECT $\left(U^{*} A * * 2\right)$ :

$\begin{array}{lllll}46.15263870 & 260.41075725 & 280.43507854 & -26.12831742\end{array}$
$+\quad 0.01639154$
0.00142323
0.00165746
0.01867684

STANDARD DISTORTION CONSTANTS (KHZ):

$\begin{array}{lll}-93.333786 & -2.693786 & -1.573786 \\ -3.413786 & -2.133786 & 12.346214\end{array}$


Table 6S: Microwave Spectrum and Spectroscopic Constants of Conformer IV of Cyclopropylmethylphosphine in Vibrationally Excited State IVb

TOTAL NUMBER OF ACCEPTED TRANSITIONS: 37

TRANSITION

OBS. FREQ. O. - C.

DISTORTION CONTRIBUTION

TOTAL

FIXED

$(\mathrm{J}, \mathrm{K}-1, \mathrm{~K}+1-->\mathrm{J}, \mathrm{K}-1, \mathrm{~K}+1)$. FREQ . DELTA

\begin{tabular}{|c|c|c|c|c|c|c|c|c|}
\hline 6 & 1 & 7 & 1 & 6 & 26622.370 & -0.232 & -0.84 & -0.11 \\
\hline 6 & 1 & 7 & 1 & 7 & 25665.840 & -0.139 & -0.60 & 0.14 \\
\hline 6 & 3 & 7 & 3 & 4 & 26177.970 & $-\odot .389$ & -0.50 & \\
\hline 6 & 4 & 7 & 4 & 3 & 26172.460 & -0.257 & -0.30 & \\
\hline 6 & 4 & 7 & 4 & 4 & 26172.460 & -0.250 & -0.30 & \\
\hline 7 & 1 & 8 & 1 & 8 & $29325.00 \odot$ & $-\odot .168$ & -0.91 & 0.20 \\
\hline 7 & 2 & 8 & 2 & 7 & 29883.690 & $\odot .347$ & $-\odot .98$ & 0.03 \\
\hline 7 & 3 & 8 & 3 & 5 & 29922.550 & 0.230 & -0.84 & \\
\hline 7 & 3 & 8 & 3 & 6 & 29919.440 & -0.136 & -0.84 & \\
\hline 7 & 4 & 8 & 4 & 4 & 29913.920 & 0.440 & -0.61 & \\
\hline 7 & 4 & 8 & 4 & 5 & 29913.920 & 0.461 & -0.61 & \\
\hline 7 & 5 & 8 & 5 & 3 & 29910.770 & 0.303 & -0.31 & -0.01 \\
\hline 7 & 5 & 8 & 5 & 4 & 29910.770 & 0.303 & -0.31 & -0.01 \\
\hline 7 & 6 & 8 & 6 & 2 & 29908.960 & -0.142 & 0.06 & -0.01 \\
\hline 7 & 6 & 8 & 6 & 3 & 29908.960 & -0.142 & 0.06 & -0.0 \\
\hline 8 & 1 & 9 & 1 & 9 & 32981.640 & 0.020 & -1.30 & 0.2 \\
\hline 8 & 2 & 9 & 2 & 8 & 33611.890 & 0.019 & -1.43 & 0. \\
\hline 8 & 3 & 9 & 3 & 6 & 33668.100 & $-\odot .085$ & -1.29 & -0.6 \\
\hline 8 & 3 & 9 & 3 & 7 & 33663.140 & -0.022 & -1.28 & \\
\hline 8 & 4 & 9 & 4 & 5 & 33655.130 & 0.160 & -1.02 & \\
\hline 8 & 4 & 9 & 4 & 6 & 33655.130 & 0.211 & -1.02 & \\
\hline 8 & 5 & 9 & 5 & 4 & 33650.440 & -0.141 & -0.68 & \\
\hline 8 & 5 & 9 & 5 & 5 & 33650.440 & -0.141 & -0.68 & \\
\hline 9 & 1 & 10 & 1 & 10 & 36635.170 & 0.050 & -1.80 & 0.38 \\
\hline 9 & 3 & 10 & 3 & 8 & 37407.670 & -0.070 & -1.85 & \\
\hline 9 & 4 & 10 & 4 & 6 & 37397.200 & $-\odot .084$ & -1.56 & \\
\hline 9 & 4 & 10 & 4 & 7 & 37397.200 & 0.026 & -1.56 & \\
\hline 9 & 5 & 10 & 5 & 5 & 37391.030 & -0.125 & -1.18 & \\
\hline 9 & 5 & 10 & 5 & 6 & 37391.030 & -0.124 & -1.18 & \\
\hline 13 & 4 & 14 & 4 & 10 & 52376.730 & -0.177 & -5.19 & -0.01 \\
\hline 13 & 410 & 14 & 4 & 11 & 52375.810 & 0.136 & -5.18 & $-\odot . \odot$ \\
\hline 13 & 5 & 14 & 5 & 9 & 52359.110 & 0.024 & -4.64 & \\
\hline 13 & 5 & 14 & 5 & 10 & 52359.110 & 0.041 & -4.64 & \\
\hline 14 & 410 & 15 & 4 & 11 & 56125.200 & 0.190 & -6.54 & $-0 . \Theta$ \\
\hline 14 & 411 & 15 & 4 & 12 & 56122.800 & -0.197 & -6.53 & $-\odot . \odot$ \\
\hline 15 & 411 & 16 & 4 & 12 & 59874.650 & -0.028 & -8.10 & $-\odot . \odot$ \\
\hline 15 & 412 & 16 & 4 & 13 & 59871.510 & 0.012 & -8.08 & $-0 . \mathrm{C}(x)+1$ \\
\hline
\end{tabular}

RMS $=0.2162 \quad$ GRAMDET.$=0.49 \mathrm{E}-\odot 3$

ROTATIONAL CONSTANTS (MHZ) :

$\begin{array}{rrrr} & 10957.070460 & 1937.396679 & 1800.505000 \\ +- & 25.648522 & 0.017584 & 0.015834\end{array}$




\begin{tabular}{|c|c|c|c|c|}
\hline$\odot .55666637$ & -2.09932259 & $26.1000 \odot \odot \odot \odot$ & $\odot .0700 \odot \odot \odot \odot$ & $1.90 \odot \odot \odot \odot \odot \odot$ \\
\hline 0.01754456 & ๑. 25047804 & $\odot .0 \odot \odot \odot \odot \odot \odot \odot$ & 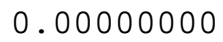 & $\odot .00000 \odot \odot \odot$ \\
\hline
\end{tabular}

SIGNIFICANT DIGITS AND CORRELATION MATRIX:

9.000

0.50215 .000

$-0.751-0.77515 .000$

$-0.718-0.114 \quad 0.555$

$\begin{array}{lllll}-0.182 & 0.402 & 0.063 & 0.099 & 5.000\end{array}$

DERIVED CONSTANTS:

KAPPA $=-0.970100$

MOMENTS OF INERTIA AND DEFECT $\left(U^{*} A * * 2\right)$ :

$46.12355573260 .85471053280 .68739055-26.29087571$

$\begin{array}{lllll}+- & 0.10796691 & 0.00236754 & 0.00246840 & 0.11106117\end{array}$

STANDARD DISTORTION CONSTANTS (KHZ):

$\begin{array}{lll}-98.229375 & -2.786665 & -1.666665 \\ -5.908020 & -2.226665 & 9.851980\end{array}$


Table 7S: Microwave Spectrum and Spectroscopic Constants of Conformer IV of Cyclopropylmethylphosphine in Vibrationally Excited State IVc

TOTAL NUMBER OF ACCEPTED TRANSITIONS: 27

TRANSITION OBS.FREQ. $0 .-\mathrm{C} . \quad \begin{gathered}\text { DISTORTION } \\ \text { TOTAL }\end{gathered} \underset{\text { FIXNTRIBUTION }}{\text { FIXED }}$

$(\mathrm{J}, \mathrm{K}-1, \mathrm{~K}+1-->\mathrm{J}, \mathrm{K}-1, \mathrm{~K}+1)$. FREQ . DELTA

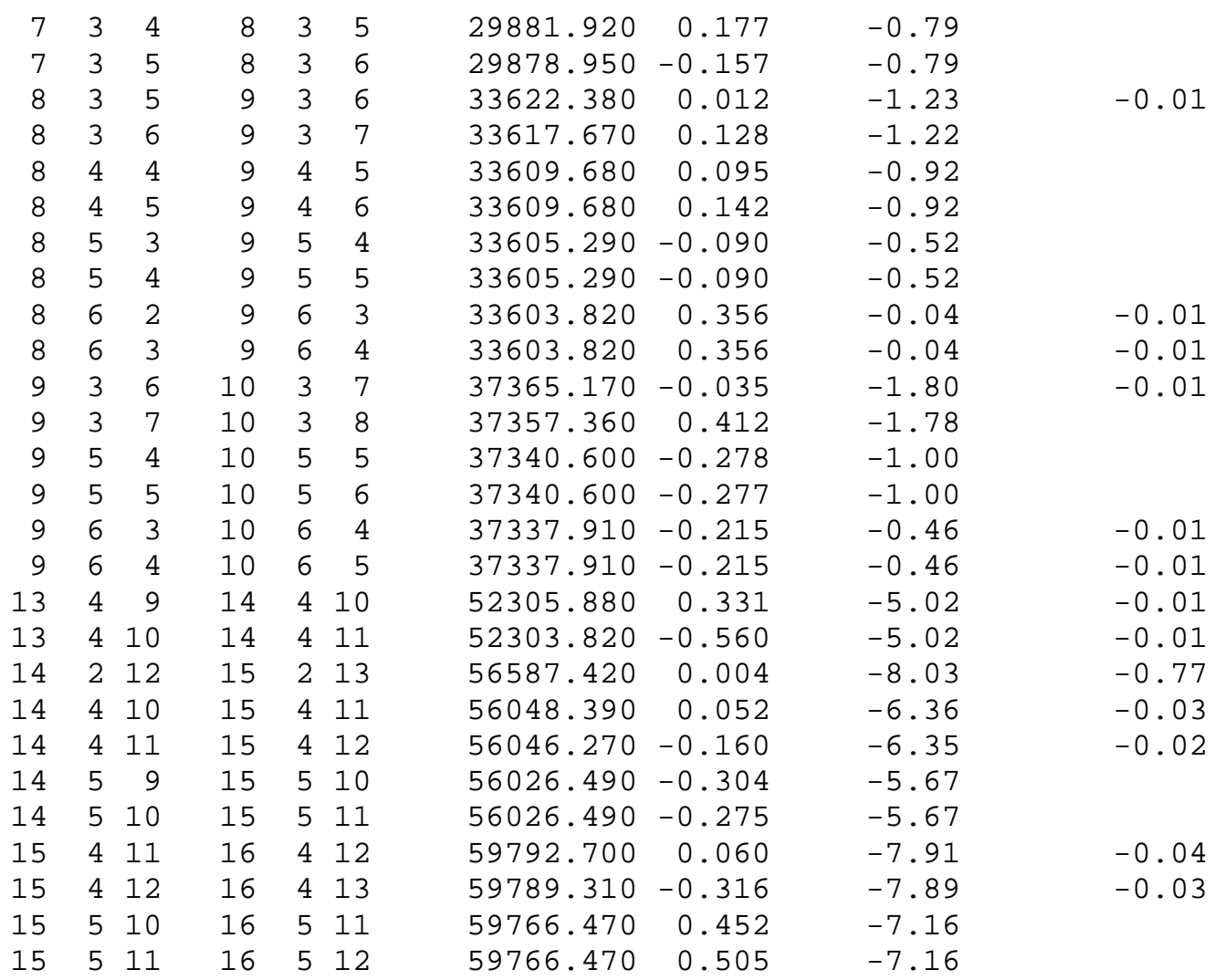

RMS $=0.2928 \quad$ GRAMDET.$=\odot .65 \mathrm{E}-\odot 3$

ROTATIONAL CONSTANTS (MHZ):
$+-$
10959.000000
0.000000
1934.001285
0.024092
1798.889596
$\odot .031521$

QUARTIC DISTORTION CONSTANTS:
$\odot .55623006$
$-2.45950990$
26.10000000
0.07000000
1.90000000
+- 0.01687640
$\odot .33881382$
0.00000000
$\odot .00000000$
0.00000000

SIGNIFICANT DIGITS AND CORRELATION MATRIX:
15.000
$-0.79314 .000$
$0.487-0.033 \quad 6.000$
$\begin{array}{llll}-0.302 & 0.687 & -0.054 & 5.000\end{array}$ 


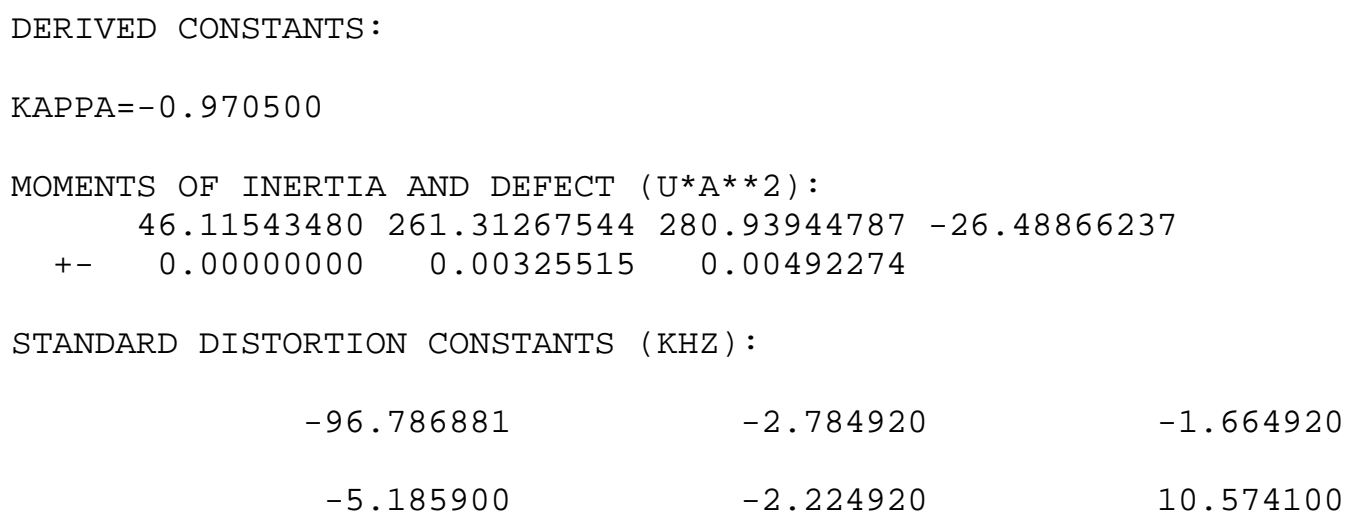


Table 8S: Microwave Spectrum and Spectroscopic Constants of Conformer IV of Cyclopropylmethylphosphine in Vibrationally Excited State IVd

TOTAL NUMBER OF ACCEPTED TRANSITIONS: 13

TRANSITION OBS.FREQ. O.-C. DISTORTION CONTRIBUTION

TOTAL FIXED

$(\mathrm{J}, \mathrm{K}-1, \mathrm{~K}+1-->\mathrm{J}, \mathrm{K}-1, \mathrm{~K}+1) . \quad \mathrm{FREQ} . \quad$ DELTA

$\begin{array}{rrrrrrrrrr}6 & 4 & 2 & 7 & 4 & 3 & 26248.910 & -0.110 & 0.80 & -0.69 \\ 6 & 4 & 3 & 7 & 4 & 4 & 26248.910 & -0.101 & 0.80 & -0.69 \\ 7 & 4 & 3 & 8 & 4 & 4 & 30000.830 & -0.018 & 0.68 & -1.03 \\ 7 & 4 & 4 & 8 & 4 & 5 & 30000.830 & 0.006 & 0.68 & -1.03 \\ 8 & 3 & 5 & 9 & 3 & 6 & 33767.020 & -0.076 & -0.39 & -1.46 \\ 8 & 3 & 6 & 9 & 3 & 7 & 33761.670 & 0.107 & -0.38 & -1.46 \\ 8 & 4 & 4 & 9 & 4 & 5 & 33753.520 & 0.049 & 0.46 & -1.46 \\ 8 & 4 & 5 & 9 & 4 & 6 & 33753.520 & 0.107 & 0.46 & -1.46 \\ 9 & 3 & 7 & 10 & 3 & 8 & 37517.350 & -0.027 & -0.80 & -2.00 \\ 14 & 4 & 10 & 15 & 4 & 11 & 56292.380 & 0.098 & -3.60 & -6.78 \\ 14 & 4 & 11 & 15 & 4 & 12 & 56289.960 & -0.032 & -3.59 & -6.77 \\ 15 & 4 & 11 & 16 & 4 & 12 & 60053.770 & -0.088 & -4.85 & -8.24 \\ 15 & 4 & 12 & 16 & 4 & 13 & 60050.280 & 0.037 & -4.83 & -8.22\end{array}$

RMS $=0.0862 \quad$ GRAMDET.$=0.44 \mathrm{E}-\odot 4$

ROTATIONAL CONSTANTS (MHZ):
$+-$ 10959.000000
1944.960776
$\odot .053787$
1803.610786
0.057191

QUARTIC DISTORTION CONSTANTS:

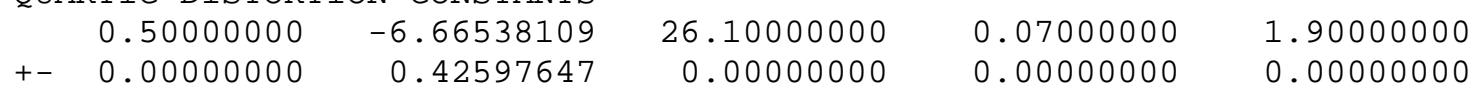

SIGNIFICANT DIGITS AND CORRELATION MATRIX:

$$
\begin{array}{rrr}
15.00 \odot & & \\
-0.974 & 15.00 \odot & \\
0.296 & -0.076 \quad 6.00 \odot
\end{array}
$$

\section{DERIVED CONSTANTS:}

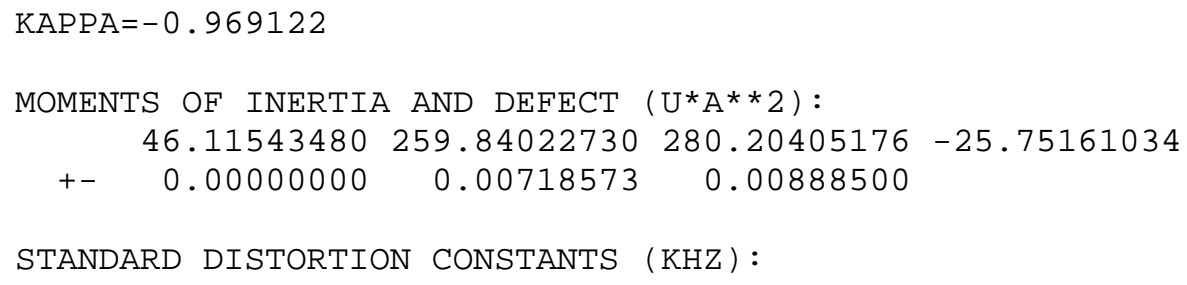

STANDARD DISTORTION CONSTANTS (KHZ):
$-79.738476$
$-2.560000$
$-1.440000$
3.450762
$-2.000000$
19. 210762 
Table 9S: Microwave Spectrum and Spectroscopic Constants of Conformer IV of Cyclopropylmethylphosphine in Vibrationally Excited State IVe

TOTAL NUMBER OF ACCEPTED TRANSITIONS: 8

TRANSITION OBS.FREQ. O.-C. DISTORTION CONTRIBUTION

TOTAL FIXED

$(\mathrm{J}, \mathrm{K}-1, \mathrm{~K}+1-->\mathrm{J}, \mathrm{K}-1, \mathrm{~K}+1)$. FREQ . DELTA

\begin{tabular}{|c|c|c|c|c|c|c|c|}
\hline 8 & 3 & 9 & 3 & 33756.100 & $\odot .057$ & -1.24 & -1.46 \\
\hline 8 & 3 & 9 & 3 & 33750.490 & $-\odot .027$ & -1.23 & -1.4 \\
\hline 9 & 3 & 10 & 3 & 37514.560 & $\odot .005$ & -1.76 & $-2 . c$ \\
\hline 9 & 3 & 10 & 3 & 37505.070 & $-\odot .031$ & -1.75 & $-2 . c$ \\
\hline 14 & 410 & 15 & 411 & 56272.910 & $\odot .161$ & -6.11 & $-6.7>0$ \\
\hline 14 & 411 & 15 & 412 & 56270.380 & -0.082 & -6.10 & 5. \\
\hline 15 & 411 & 16 & 412 & 60032.940 & $-\odot .078$ & -7.53 & -8 \\
\hline 15 & 412 & 16 & 413 & 60029.410 & $\odot .003$ & -7.51 & -8 \\
\hline
\end{tabular}

RMS $=0.0937 \quad$ GRAMDET.$=0.82 \mathrm{E}-05$

ROTATIONAL CONSTANTS (MHZ):
$+-$
10959.000000
1944.364466
1803.076881
0.198578

QUARTIC DISTORTION CONSTANTS:
0.50000000
$-1.39753283$
$+-0.00000000$
0.65922052
26.10000000
$\odot .07000000$
1.90000000
$\odot .00000000$
0.00000000
0.00000000

SIGNIFICANT DIGITS AND CORRELATION MATRIX:

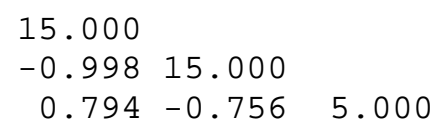

DERIVED CONSTANTS:

KAPPA $=-0.969137$

MOMENTS OF INERTIA AND DEFECT $(U * A * * 2)$ :

$\begin{array}{lllll}46.11543480 & 259.91991665 & 280.28702232 & -25.74832914\end{array}$

+- $\quad 0.000000000 .02525603 \quad 0.03086881$

STANDARD DISTORTION CONSTANTS (KHZ):
$-100.809869$
$-2.560000$
$-1.440000$
$-7.084934$
$-2.000000$
8.675066 
Table 10S: Microwave Spectrum and Spectroscopic Constants of the Ground Vibrational State of Cyclopropylmethylphosphine, Conformer V

TOTAL NUMBER OF ACCEPTED TRANSITIONS: 161

TRANSITION

$(\mathrm{J}, \mathrm{K}-1, \mathrm{~K}+1-->\mathrm{J}, \mathrm{K}-1, \mathrm{~K}+1) . \quad$ FREQ. $\quad$ DELTA

\author{
OBS . FREQ. $0 .-\mathrm{C}$. \\ DISTORTION CONTRIBUTION \\ TOTAL SEXTIC
}

$26960.380 \quad 0.253$

$27742.080-0.168$

$30371.160 \quad 0.476$

$24713.120-0.070$

51899.690

$\odot .045$

$28756.070 \quad 0.168$

$37029.810-0.033$

$30912.650-0.045$

$30748.130-0.113$

$29382.390-0.773$

$30798.750-0.176$

$30794.910 \quad 0.044$

$30787.490-0.070$

$30787.490-0.034$

30784.100

0.057

30784.100

0.057

$27963.250-0.196$

$30091.720 \quad 0.107$

$32237.380-0.093$

$30881.890 \quad 0.068$

$36509.520 \quad 0.045$

$25239.710-0.121$

$49972.200-0.263$

$22727.570 \quad 0.063$

32709.040

29968.130

0.029

$\odot .295$

17671.620

0.005

$53192.830-0.145$

50537.820

0.079

$22470.550 \quad 0.004$

$49886.780-0.094$

$33746.130-0.062$

$50122.020 \quad 0.118$

$50074.360 \quad 0.036$

$45030.740 \quad 0.042$

$50056.290-0.159$

50055.440

50038.830

0.226

0.055

$50038.830 \quad 0.071$

$50030.630-0.077$

$50030.630-0.077$

54490.570

0.074

22300.880

$-0.004$

$53702.730 \quad 0.077$

$34865.450 \quad 0.009$

$54001.100-0.026$
$-0.08$

$-0.17$

$-0.17$

0.77

$-0.23$

$-0.08$

$-0.50$

$-1.34$

$-1.20$

$-0.11$

$-0.83$

$-0.82$

$-0.24$

$-0.24$

0.52

0.52

$-2.94$

$-0.21$

$-3.95$

$-0.41$

$-5.13$

$-9.61$

$-2.12$

2.68

$-1.24$

$-12.26$

$-5.53$

$-2.85$

$-7.08$

2.85

$-5.90$

$-1.92$

$-5.72$

$-5.58$

1.70

$-4.62$

$-4.62$

$-3.37$

$-3.37$

$-1.86$

$-1.86$

$-9.01$

2.82

$-7.43$

$-2.83$

$-7.41$
0.01 


\begin{tabular}{|c|c|c|c|c|c|c|c|}
\hline 13 & 311 & 14 & 312 & 53932.420 & 0.038 & -7.19 & \\
\hline 13 & 49 & 14 & 410 & 53914.470 & -0.101 & -6.17 & \\
\hline 13 & 410 & 14 & 411 & 53912.420 & -0.058 & -6.16 & \\
\hline 13 & 58 & 14 & 59 & 53892.090 & 0.101 & -4.80 & \\
\hline 13 & 5 & 14 & 510 & 53892.090 & 0.133 & -4.80 & \\
\hline 14 & 114 & 15 & ๑ 15 & 53380.690 & $\odot .154$ & -11.43 & \\
\hline 14 & 213 & 15 & 114 & 39553.260 & -0.022 & -18.84 & \\
\hline 15 & 015 & 15 & 114 & 21038.180 & -0.007 & -10.24 & 0.01 \\
\hline 15 & 114 & 15 & 213 & 22234.170 & $\odot .032$ & 2.49 & \\
\hline 15 & 115 & 15 & 214 & 36066.140 & 0.028 & -4.01 & \\
\hline 14 & 311 & 15 & 312 & 57886.950 & -0.168 & -9.41 & \\
\hline 14 & 312 & 15 & 313 & 57790.500 & -0.036 & -9.05 & \\
\hline 14 & 410 & 15 & 411 & 57774.550 & -0.003 & -7.98 & \\
\hline 14 & 411 & 15 & 412 & 57771.340 & 0.203 & -7.96 & \\
\hline 15 & 313 & 15 & 412 & 61889.930 & 0.079 & 3.49 & \\
\hline 15 & 115 & 16 & 016 & 57509.060 & $\odot .071$ & -13.38 & \\
\hline 15 & 214 & 16 & 115 & 44396.980 & -0.029 & -22.78 & \\
\hline 16 & $\odot 16$ & 16 & 115 & 22954.140 & 0.008 & -13.41 & 0.01 \\
\hline 15 & 313 & 16 & 214 & 21144.150 & -0.207 & -23.07 & 0.01 \\
\hline 16 & 115 & 16 & 214 & 22284.620 & 0.060 & 1.78 & 0.01 \\
\hline 16 & 116 & 16 & 215 & 37347.120 & -0.081 & -5.48 & \\
\hline 17 & 017 & 17 & 116 & 25016.610 & -0.159 & -17.15 & 0.01 \\
\hline 16 & 314 & 17 & 215 & 25863.280 & -0.070 & -28.59 & 0.01 \\
\hline 17 & 314 & 17 & 413 & 61278.930 & 0.078 & 7.91 & \\
\hline 17 & 315 & 17 & 414 & 61859.850 & 0.039 & 5.80 & \\
\hline 17 & 216 & 18 & 117 & 54145.360 & 0.070 & -31.93 & 0.01 \\
\hline 18 & $\odot 18$ & 18 & 117 & 27215.690 & -0.231 & -21.48 & 0.01 \\
\hline 18 & 117 & 18 & 216 & 22787.080 & $\odot .006$ & -1.20 & $\odot .01$ \\
\hline 18 & 118 & 18 & 217 & 40144.860 & 0.152 & -9.45 & \\
\hline 18 & 216 & 18 & 315 & 39515.610 & 0.072 & 15.17 & -0.01 \\
\hline 18 & 315 & 18 & 414 & 61052.250 & 0.039 & 10.16 & \\
\hline 18 & 316 & 18 & 415 & 61853.860 & 0.006 & 6.99 & \\
\hline 19 & $\odot 19$ & 19 & 118 & 29538.600 & -0.166 & -26.38 & 0.02 \\
\hline 18 & 316 & 19 & 217 & 35596.150 & 0.024 & -42.10 & 0.01 \\
\hline 19 & 118 & 19 & 217 & 23261.140 & 0.040 & -3.71 & $\odot . \odot 2$ \\
\hline 18 & 315 & 19 & 218 & 25174.660 & -0.223 & -16.59 & -0.03 \\
\hline 19 & 119 & 19 & 218 & 41657.270 & $\odot .076$ & -12.01 & \\
\hline 19 & 217 & 19 & 316 & 38755.930 & -0.186 & 17.68 & -0.01 \\
\hline 19 & 316 & 19 & 415 & 60773.640 & -0.118 & 12.80 & -0.01 \\
\hline 19 & 317 & 19 & 416 & 61857.730 & -0.042 & 8.17 & 0.01 \\
\hline 20 & $\odot 20$ & 20 & 119 & 31970.340 & -0.023 & -31.82 & $\odot .02$ \\
\hline 20 & 119 & 20 & 218 & 23896.330 & -0.033 & -7.06 & 0.03 \\
\hline 20 & 218 & 20 & $\begin{array}{ll}3 & 17\end{array}$ & 37984.330 & 0.022 & 20.12 & -0.01 \\
\hline 20 & 219 & 20 & 318 & 47783.690 & 0.289 & -0.62 & 0.02 \\
\hline 20 & 317 & 20 & 416 & $6 \odot 436.070$ & -0.113 & 15.92 & -0.01 \\
\hline 20 & 318 & 20 & 417 & 61874.950 & -0.004 & 9.30 & 0.01 \\
\hline 21 & $\odot 21$ & 21 & 120 & 34494.220 & -0.047 & -37.74 & 0.02 \\
\hline 21 & 120 & 21 & 219 & 24701.200 & 0.189 & -11.41 & 0.04 \\
\hline 21 & 121 & 21 & 220 & 44897.270 & 0.260 & -18.45 & -0.01 \\
\hline 21 & 219 & 21 & 318 & 37219.530 & $-\odot .037$ & 22.37 & -0.01 \\
\hline 22 & $\odot 22$ & 22 & 121 & 37093.310 & 0.108 & -44.09 & $\odot .01$ \\
\hline 22 & 121 & 22 & 220 & 25681.790 & -0.157 & -16.87 & 0.05 \\
\hline 22 & 220 & 22 & $\begin{array}{ll}3 & 19\end{array}$ & 36482.490 & -0.113 & 24.23 & -0.01 \\
\hline 22 & 319 & 22 & 418 & 59556.070 & 0.122 & 23.89 & $-\odot .02$ \\
\hline 22 & 320 & 22 & 419 & 61964.410 & -0.110 & 11.27 & 0.02 \\
\hline 23 & 122 & 23 & 221 & 26844.410 & -0.159 & -23.59 & 0.07 \\
\hline 23 & 123 & 23 & 222 & 48402.530 & -0.054 & -26.79 & $-\odot .02$ \\
\hline
\end{tabular}




\begin{tabular}{|c|c|c|c|c|c|c|c|}
\hline 22 & 418 & 23 & 321 & 26480.240 & -0.050 & -45.11 & -0.04 \\
\hline 23 & 320 & 23 & 419 & 59001.520 & -0.072 & 28.89 & -0.02 \\
\hline 24 & 123 & 24 & 222 & 28192.290 & -0.136 & -31.68 & 0.08 \\
\hline 24 & 124 & 24 & 223 & 50246.450 & 0.185 & -31.72 & -0.03 \\
\hline 24 & 222 & 24 & 321 & 35177.270 & -0.067 & 26.04 & \\
\hline 23 & 419 & 24 & 322 & 30171.500 & 0.118 & -50.01 & -0.06 \\
\hline 24 & 223 & 24 & 322 & 50836.180 & 0.388 & -9.99 & 0.05 \\
\hline 24 & 321 & 24 & 420 & 58365.970 & 0.176 & 34.61 & -0.03 \\
\hline 25 & 025 & 25 & 124 & 45169.540 & 0.208 & -64.92 & -0.01 \\
\hline 25 & 124 & 25 & 223 & 29726.810 & -0.014 & -41.22 & 0.10 \\
\hline 25 & 125 & 25 & 224 & 52145.260 & -0.210 & -37.17 & -0.04 \\
\hline 25 & 223 & 25 & 322 & 34651.010 & -0.095 & 25.54 & 0.01 \\
\hline 25 & 224 & 25 & 323 & 51795.200 & $\odot .298$ & -13.83 & 0.05 \\
\hline 25 & 322 & 25 & 421 & 57647.200 & -0.105 & 41.07 & $-\odot .04$ \\
\hline 26 & 026 & 26 & 125 & 47902.340 & -0.233 & -72.24 & -0.02 \\
\hline 26 & 125 & 26 & 224 & 31446.470 & 0.016 & -52.26 & 0.13 \\
\hline 26 & 126 & 26 & 225 & 54095.920 & -0.326 & -43.15 & -0.06 \\
\hline 26 & 224 & 26 & 323 & 34235.620 & -0.107 & 23.80 & 0.03 \\
\hline 26 & 323 & 26 & 422 & 56847.660 & 0.006 & 48.23 & -0.05 \\
\hline 27 & 027 & 27 & 126 & 50634.690 & -0.063 & -79.67 & -0.04 \\
\hline 27 & 126 & 27 & 225 & 33347.030 & -0.077 & -64.80 & 0.15 \\
\hline 27 & 127 & 27 & 226 & 56094.550 & -0.037 & -49.67 & -0.08 \\
\hline 27 & 225 & 27 & 324 & 33949.270 & -0.161 & 20.58 & 0.05 \\
\hline 27 & 226 & 27 & 325 & 53956.670 & 0.085 & -23.72 & 0.07 \\
\hline 27 & 324 & 27 & 423 & 55971.320 & -0.192 & 55.98 & -0.06 \\
\hline 28 & 028 & 28 & 127 & 53356.020 & -0.104 & -87.21 & -0.07 \\
\hline 28 & 127 & 28 & 226 & 35421.560 & 0.035 & -78.80 & 0.18 \\
\hline 28 & 226 & 28 & 325 & 33808.640 & -0.116 & 15.64 & 0.07 \\
\hline 28 & 325 & 28 & 424 & 55026.610 & -0.296 & 64.16 & -0.07 \\
\hline 29 & 128 & 29 & 227 & 37659.390 & -0.038 & -94.15 & 0.22 \\
\hline 29 & 227 & 29 & 326 & 33828.300 & -0.144 & 8.74 & 0.10 \\
\hline 29 & 228 & 29 & 327 & 56443.110 & 0.117 & -36.89 & 0.09 \\
\hline 30 & 129 & 30 & 228 & 40047.680 & -0.028 & -110.70 & 0.25 \\
\hline 30 & 228 & 30 & 327 & 34021.340 & -0.083 & -0.36 & 0.14 \\
\hline 30 & 229 & 30 & 328 & 57806.240 & 0.159 & -44.81 & 0.10 \\
\hline 31 & 130 & 31 & 229 & 42570.430 & -0.369 & -128.24 & 0.28 \\
\hline 31 & 229 & 31 & 328 & 34398.720 & -0.122 & -11.89 & 0.19 \\
\hline 32 & 230 & 32 & 329 & 34970.000 & -0.108 & -26.07 & 0.25 \\
\hline 33 & 132 & 33 & 231 & 47950.340 & 0.370 & -165.34 & 0.34 \\
\hline 33 & 231 & 33 & 330 & 35742.790 & -0.092 & -43.13 & 0.32 \\
\hline 34 & 133 & 34 & 232 & 50767.610 & $\odot .002$ & -184.38 & 0.36 \\
\hline 34 & 232 & 34 & 331 & 36723.010 & 0.019 & -63.23 & 0.41 \\
\hline 35 & 134 & 35 & 233 & 53644.590 & 0.055 & -203.40 & 0.38 \\
\hline 35 & 233 & 35 & 332 & 37914.160 & -0.100 & -86.51 & 0.50 \\
\hline 36 & 135 & 36 & 234 & 56562.040 & 0.176 & -222.19 & 0.39 \\
\hline 36 & 234 & 36 & 333 & 39318.210 & -0.062 & -113.03 & 0.62 \\
\hline 37 & 136 & 37 & 235 & 59502.040 & 0.038 & -240.56 & 0.40 \\
\hline 38 & 236 & 38 & 335 & 42757.750 & -0.289 & -175.64 & 0.88 \\
\hline 39 & 237 & 39 & 336 & 44783.420 & $-\odot .011$ & -211.37 & 1.03 \\
\hline 41 & 239 & 41 & 338 & 49396.890 & 0.088 & -290.00 & 1.37 \\
\hline 42 & 240 & 42 & 339 & 51956.690 & -0.004 & -331.91 & 1.55 \\
\hline 43 & 241 & 43 & 340 & 54662.380 & -0.081 & -374.79 & 1.73 \\
\hline 43 & 340 & 43 & $4 \quad 39$ & 45027.650 & 0.283 & -21.57 & 0.88 \\
\hline 44 & 242 & 44 & 341 & 57494.390 & -0.055 & -417.98 & 1.91 \\
\hline 45 & 243 & 45 & 342 & 60431.810 & 0.090 & -460.84 & 2.08 \\
\hline 51 & 447 & 51 & 546 & 55883.200 & 0.046 & 217.18 & 0.51 \\
\hline 52 & 448 & 52 & 547 & 55419.620 & 0.063 & 173.66 & 0.94 \\
\hline
\end{tabular}




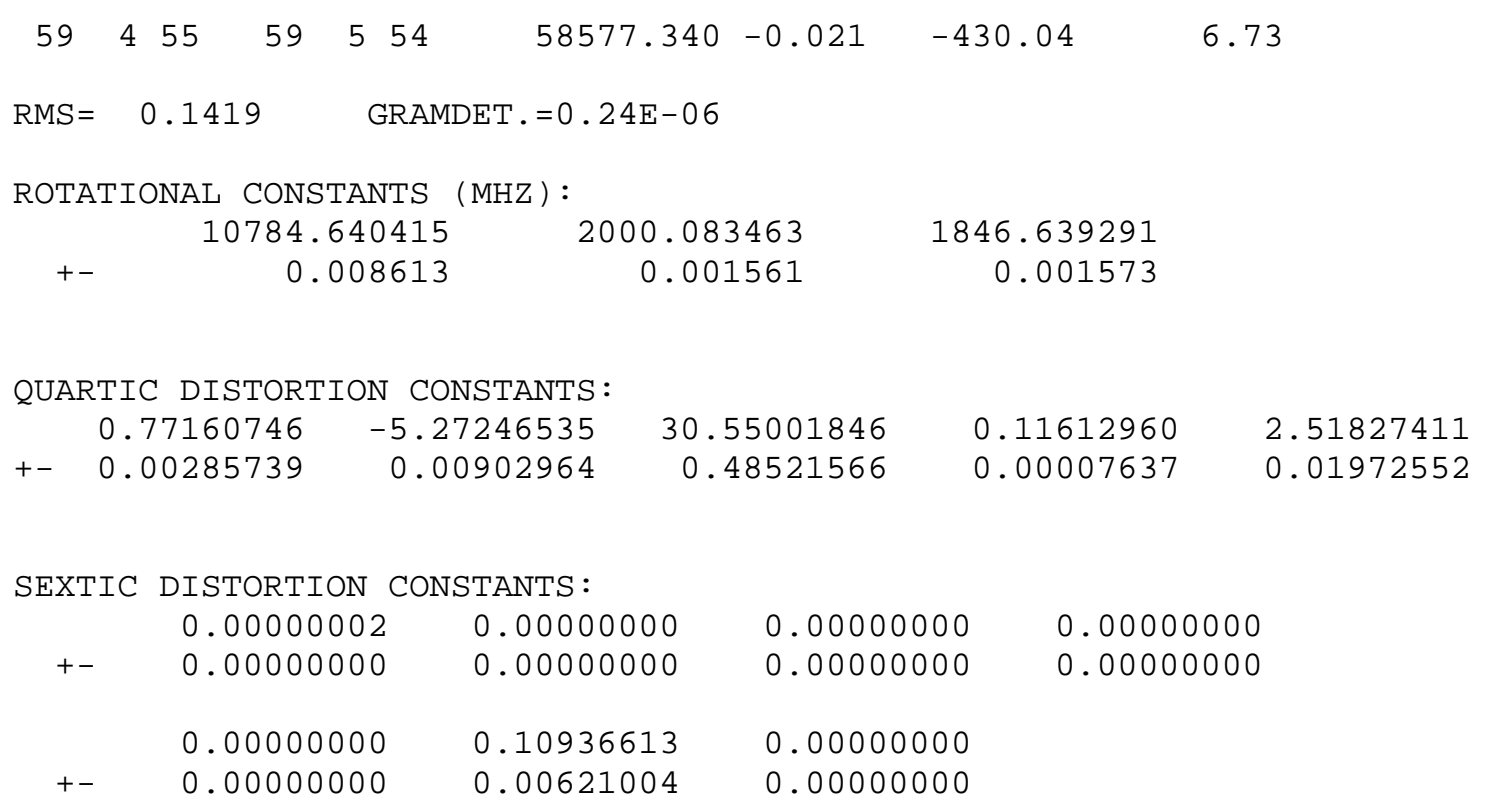

\section{SIGNIFICANT DIGITS AND CORRELATION MATRIX:}

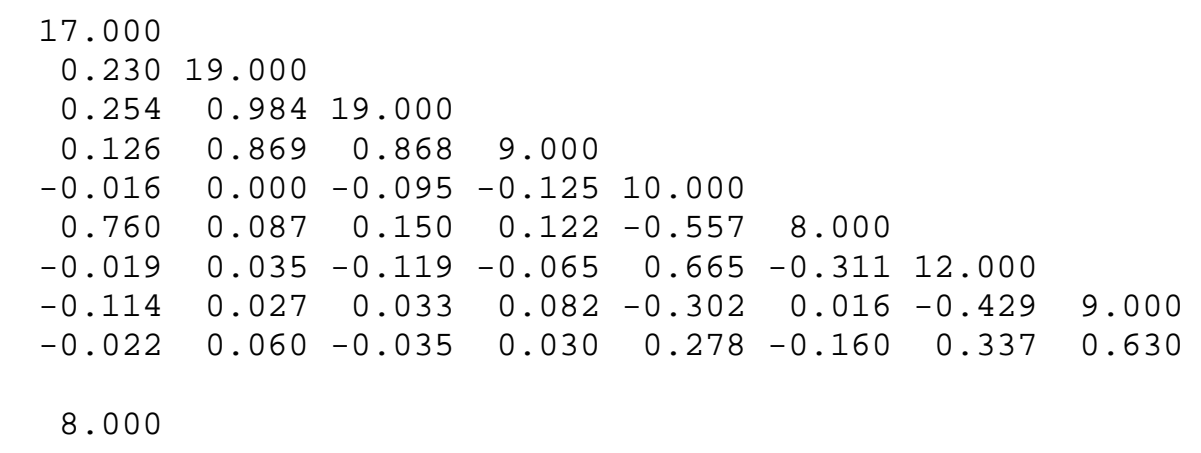

\section{DERIVED CONSTANTS:}

KAPPA $=-0.965665$

MOMENTS OF INERTIA AND DEFECT $\left(U^{*} A^{* *} 2\right)$ : $46.86100144252 .67898035273 .67502269-25.86495910$

$\begin{array}{lllll}+- & 0.00003742 & 0.00019717 & 0.00023317 & 0.00005629\end{array}$

STANDARD DISTORTION CONSTANTS (KHZ):

$\begin{array}{rrr}-104.196642 & -4.015467 & -2.157393 \\ -3.079114 & -3.086430 & 17.996116\end{array}$


TABLE 11S: Geometries ${ }^{a}$ of two conformers of cyclopropylmethylphosphine from $a b$ initio calculations at the MP2/aug-cc-pVDZ level of theory

\begin{tabular}{|c|c|c|}
\hline Molecular dimension & Conformer I & Conformer II \\
\hline \multicolumn{3}{|l|}{ Bond Lengths: } \\
\hline$r\left(\mathrm{PH}_{1}\right)$ & 141.3 & 141.1 \\
\hline$r\left(\mathrm{PH}_{2}\right)$ & 141.3 & 141.3 \\
\hline$r\left(\mathrm{PC}_{1}\right)$ & 185.9 & 186.9 \\
\hline$r\left(\mathrm{C}_{1} \mathrm{H}_{3}\right)$ & 109.4 & 109.0 \\
\hline$r\left(\mathrm{C}_{1} \mathrm{H}_{4}\right)$ & 109.4 & 109.3 \\
\hline$r\left(\mathrm{C}_{1} \mathrm{C}_{2}\right)$ & 151.3 & 151.2 \\
\hline$r\left(\mathrm{C}_{2} \mathrm{H}_{5}\right)$ & 108.4 & 108.3 \\
\hline$r\left(\mathrm{C}_{2} \mathrm{C}_{3}\right)$ & 150.3 & 150.6 \\
\hline$r\left(\mathrm{C}_{2} \mathrm{C}_{4}\right)$ & 150.3 & 150.4 \\
\hline$r\left(\mathrm{C}_{3} \mathrm{H}_{6}\right)$ & 107.9 & 108.0 \\
\hline$r\left(\mathrm{C}_{3} \mathrm{H}_{7}\right)$ & 108.0 & 108.1 \\
\hline$r\left(\mathrm{C}_{3} \mathrm{C}_{4}\right)$ & 151.0 & 150.9 \\
\hline$r\left(\mathrm{C}_{4} \mathrm{H}_{8}\right)$ & 107.9 & 107.9 \\
\hline$r\left(\mathrm{C}_{4} \mathrm{H}_{9}\right)$ & 108.0 & 108.1 \\
\hline \multicolumn{3}{|l|}{ Bond Angles: } \\
\hline$\angle\left(\mathrm{H}_{1} \mathrm{PC}_{1}\right)$ & 99.0 & 98.0 \\
\hline$\angle\left(\mathrm{H}_{2} \mathrm{P}, \mathrm{C}_{1}\right)$ & 99.0 & 96.0 \\
\hline$\angle\left(\mathrm{P}_{1} \mathrm{C}_{1} \mathrm{C}_{2}\right)$ & 121.4 & 113.9 \\
\hline$\angle\left(\mathrm{H}_{3} \mathrm{C}_{1} \mathrm{C}_{2}\right)$ & 109.4 & 110.1 \\
\hline$\angle\left(\mathrm{H}_{4} \mathrm{C}_{1} \mathrm{C}_{2}\right)$ & 109.4 & 109.4 \\
\hline$\angle\left(\mathrm{C}_{1} \mathrm{C}_{2} \mathrm{C}_{3}\right)$ & 123.3 & 122.0 \\
\hline$\angle\left(\mathrm{C}_{1} \mathrm{C}_{2} \mathrm{C}_{4}\right)$ & 123.3 & 121.5 \\
\hline$\angle\left(\mathrm{H}_{5} \mathrm{C}_{2} \mathrm{C}_{1}\right)$ & 112.4 & 113.6 \\
\hline$\angle\left(\mathrm{H}_{6} \mathrm{C}_{3} \mathrm{C}_{2}\right)$ & 117.9 & 118.1 \\
\hline$\angle\left(\mathrm{H}_{7} \mathrm{C}_{3} \mathrm{C}_{2}\right)$ & 117.8 & 117.9 \\
\hline$\angle\left(\mathrm{H}_{8} \mathrm{C}_{4} \mathrm{C}_{2}\right)$ & 117.9 & 117.8 \\
\hline$\angle\left(\mathrm{H}_{9} \mathrm{C}_{4} \mathrm{C}_{2}\right)$ & 117.8 & 117.5 \\
\hline \multicolumn{3}{|l|}{ Dihedral Angles: } \\
\hline$\angle\left(\mathrm{H}_{1} \mathrm{PC}_{1}, \mathrm{C}_{2}\right)$ & 47.7 & -86.8 \\
\hline$\angle\left(\mathrm{H}_{2} \mathrm{PC}_{1}, \mathrm{C}_{2}\right)$ & -47.7 & 178.6 \\
\hline$\angle\left(\mathrm{PC}_{1} \mathrm{C}_{2}, \mathrm{C}_{3}\right)$ & -37.0 & 30.2 \\
\hline$\angle\left(\mathrm{PC}_{1} \mathrm{C}_{2}, \mathrm{C}_{4}\right)$ & 37.0 & -42.1 \\
\hline$\angle\left(\mathrm{H}_{3} \mathrm{C}_{1} \mathrm{C}_{2}, \mathrm{C}_{3}\right)$ & 85.2 & -93.8 \\
\hline$\angle\left(\mathrm{H}_{4} \mathrm{C}_{1} \mathrm{C}_{2}, \mathrm{C}_{4}\right)$ & -85.2 & 76.1 \\
\hline$\angle\left(\mathrm{H}_{5} \mathrm{C}_{2} \mathrm{C}_{1}, \mathrm{P}\right)$ & 180.0 & 174.4 \\
\hline$\angle\left(\mathrm{H}_{6} \mathrm{C}_{3} \mathrm{C}_{2}, \mathrm{C}_{1}\right)$ & 6.1 & 141.2 \\
\hline$\angle\left(\mathrm{H}_{7} \mathrm{C}_{3} \mathrm{C}_{2}, \mathrm{C}_{1}\right)$ & -139.4 & -4.2 \\
\hline$\angle\left(\mathrm{H}_{8} \mathrm{C}_{3} \mathrm{C}_{2}, \mathrm{C}_{1}\right)$ & -6.1 & -140.6 \\
\hline$\angle\left(\mathrm{H}_{9} \mathrm{C}_{3} \mathrm{C}_{2}, \mathrm{C}_{1}\right)$ & 139.4 & 4.8 \\
\hline
\end{tabular}

${ }^{a}$ Distances in pm and angles in degrees. 